\title{
A Novel and Efficient Method to Extract Blood Vessels from Retinal Images
}

Manuscrit reçu le 13 juin 2016 et accepté le 10 juillet 2016

\author{
Saleh Shahbeig ${ }^{1, *}$, Mohammad Sadegh Helfroush ${ }^{2}$ \\ ${ }^{1}$ Young Researchers and Elite Club, Najafabad Branch, Islamic Azad University, Najafabad, Iran \\ ${ }^{2}$ Department of Electrical and Electronics Engineering, Shiraz University of Technology, Shiraz, Iran \\ * Corresponding author: saleh_shahbeig@yahoo.com
}

\begin{abstract}
The retinal images contain vital features which are important in the analysis of the retina for various medical applications or human identification. In this manuscript, an Adaptive Weighted Morphological Functor (AWMF) method is proposed for the extraction of blood vessels. The method considers the dispersion of the blood vessel in various directions in the retinal images with prior knowledge of the approximate direction of the edges. The proposed blood vessels extraction algorithm, using principle component analysis (PCA) technique and estimation of the brightness and contrast of the background, effectively eliminates interference of regions with intense brightness structure from the background light distribution. Then the method selects a proper way to modify the brightness distribution of the background, regardless of the destructive effect of brightness structures such as optical disc (OD). The experimental results indicate that the proposed algorithm achieves an accuracy rate of $97.11 \%$ for the blood vessels extraction on the famous databases, DRIVE.
\end{abstract}

Keywords - retinal images, segmentation, blood vessels, adaptive weighted morphological functors, principle component analysis.

\section{INTRODUCTION}

Retina is the most important inner part of the eye. It covers the whole back part of the eye and the light receptors are distributed on it. By extracting the proper features from the retinal images, we can detect eye diseases in the retinal images or achieve useful and effective measures such as automatic recognition in human recognition systems. The blood vessels are the important parts of the retinal images, consisting of arteries and arterioles. Checking the obtained changes in retinal images at a certain time interval can help the physician to diagnose the disease. Applications of the retinal images 
are diagnosing the progress of some cardiovascular diseases, diagnosing the region with no blood vessels (Macula), using such images in helping automatic laser surgery on eye, and using the images in biometric applications, etc. On the other hand, extracting the retinal blood vessels is manually accompolished in some cases by a physician, which is a difficult and time-consuming task and is accompanied by high mistakes due to much dependence on the physician's skill level. Therefore, the exact extraction of the blood vessels from the retinal images necessitates an algorithm and instrument which reduce the dependency on the physician's skill level and eliminate the error factors. In the most common methods used to extract the blood vessels, tracking-based [1], classifying-based [2] and window-based [3] methods can be referenced. Because of the variability of light reflection coefficients in different parts of the retina layer and the defects which exist in imaging systems, there occurs very non-uniform illumination in the retinal images, which impairs modeling the blood vessels in window-based methods and tracking in tracking-based methods. Since all connected regions in retinal images are classified by a low-level algorithm in classifying-based methods, the pixels related to the blood vessels cannot be classified carefully due to the intrinsic noise in the retinal images and oscillating changes in image illumination. As a result, for extraction of blood vessels with high accuracy, we need an effective algorithm. The most proposed methods to extract the blood vessels from retinal images have relatively high computational complexity. To overcome these limitations and in order to increase the system's speed and accuracy, this paper proposes a novel algorithm to extract the blood vessels, fast, easily and highly accurate in the retinal images.

In this paper, by local estimating the illumination of the background and contrast distribution in the retinal images [4], the illumination of the retinal image is uniformed. Since the pre-processing phase plays an important role in the final extraction results, estimating locally the illumination of the background and contrast distribution in the retinal images will have a noticeable effect on improving the accuracy of the final edge image. To ensure that the OD region in the retinal images does not have adverse effects on the uniform illumination of the background, using PCA technique on the three RGB channels of the original retinal images before applying the first sampling process has been proposed in this paper. The PCA technique in the pre-processing procedure can increase speed of our algorithm more than by two times in addition to stability of the proposed algorithm performance.

Since the blood vessels are scattered in different directions, for the first time in this article, Adaptive Weighted Morphological Functors (AWMF) are introduced for extracting blood vessels from retinal images. AWMF technique causes the blood vessels with high accuracy and connectivity to be extracted. Finally, using the connected component analysis (CCA) with 4 and 8 neighborhoods 
of the pixels, respectively in two steps and by applying the adaptive filter on the created components locally [8], the frills in the image are removed and the extracted blood vessels are obtained.

The rest of the paper is organized as following:

In section 2, the proposed algorithm is described in detail. In section 3, the experimental results are presented and discussed. Our conclusions are presented in the section 4.

\section{THE PROPOSED ALGORITHM}

Fig. 1 shows a block diagram of the proposed retinal image blood vessel extraction algorithm.

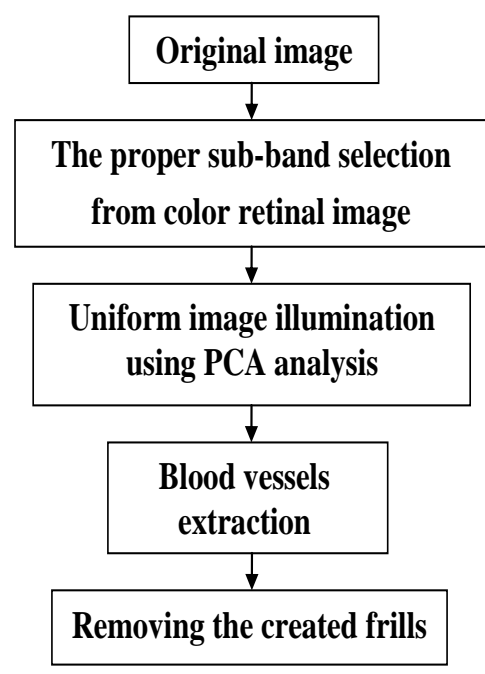

Fig. 1: Block diagram of the proposed algorithm.

\subsection{Pre-processing}

As the sub-band $\mathrm{G}$ from the colored retinal images has the highest contrast between the sub-bands, this enhancement is achieved on the sub-band G (Green sub-band). Since the non-uniformity of illumination in the retinal images generates the frills in the final edge image, so it is necessary to uniform the image illumination. Locally estimation of the illumination and the contrast distribution in the retinal images is used to uniform the illumination of the images [4] in this paper. This stage produces an image with a uniformed and darker background and brighter gray level for the blood vessels. 


\subsubsection{Smoothing illumination}

In this section, the existing illumination changes in the background of the retinal images are corrected by estimating the illumination of the background and contrast distribution [4]. The enhanced image of $U(x, y)$ is formulated as follows.

$U(x, y)=\frac{I(x, y)-k_{1} \cdot L(x, y)}{k_{2} \cdot C(x, y)}$

where $I$ is the original image, $C$ and $L$ are the contrast and illumination deviations of the pixel located in location $(x, y)$, respectively. $k_{1}$ and $k_{2}$ are constant coefficients. $L$ and $C$ are estimated through sampling the original image. Due to the circular structure of the OD region in the retinal images, we use a non-uniform sampling grid shown in Fig. 2(a).

One advantage of this sampling grid is less computational complexity of the proposed algorithm. As shown in Fig. 2(a), sampling in this grid is coarse in the central region and dense in the border. As the sub-band $\mathrm{G}$ from the colored retinal images has the highest contrast between the sub-bands, this enhancement is performed on the sub-band G. In order to effectively correct the destructive illumination changes in the retinal images, the components $C$ and $L$ are estimated only in the background pixels. Therefore, the first step is to segment the image pixels into the background and foreground pixels. A $W \times W$ sized window, large enough to encompass the components of the image and the background, is dedicated to each point of sampling as shown in Fig. 2(a). Then, for each point, the local mean, $\mu$, and the standard deviation, $\sigma$, are calculated. We use the bi-cubic interpolation to calculate the mean and the standard deviation of the remaining pixels in the retinal images. To recognize the pixels in the background, Mahalanobis distance is used as,

$$
D(x, y)=\left|\frac{I(x, y)-\mu(x, y)}{\sigma(x, y)}\right|
$$

According to above relation, the pixels whose Mahalanobis distance $(D(x, y))$ from $\mu$ is less than the local threshold $t$ is labeled as background. The quantity of local threshold $t$ in this section is defined as:

$$
t=\mu-\alpha \cdot \sigma
$$


where $\mu$ and $\sigma$ are the local mean and the standard deviation, respectively and $\alpha$ is a constant value that controls the amount of the threshold $t . t$ is a local threshold which is variable for all pixels in the retinal image. Additional details about the performance of this function are available in [5].

The retinal components that have the intense illumination changes such as the OD region can influence the illumination of the background. Using the PCA technique on the three RGB channels of the original retinal images before applying the proposed sampling grid, we localize the OD region primary to ensure that the OD region is not to be taken into account in this estimation. The PCA technique in the pre-processing procedure can increase speed of our algorithm more than two times as well as stability of the proposed algorithm performance. Additional details about the performance of the PCA technique are available in [5]. The final improved image is obtained by applying the Eq. (1) on the considered retinal image. The effects of pre-processing stage on retinal images are shown in Fig. 2. It is obvious that in Fig. 2(e) the illumination in the surrounding region of the optic disc (OD) has not been modified significantly compared to that in Fig. 2(g) because, the OD component is merged in the background and effects on the estimation process (Fig. 2(f)).

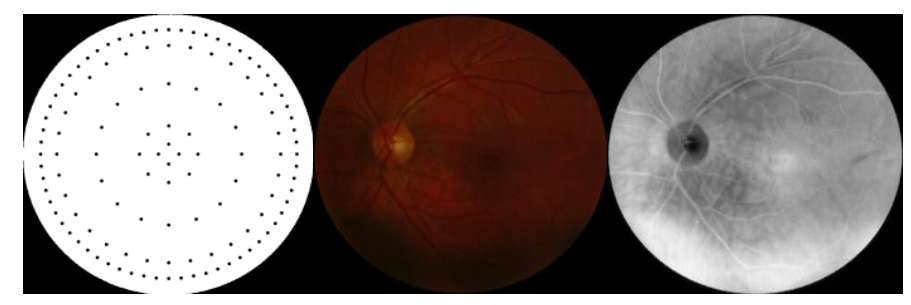

(a)

(b)

(c)

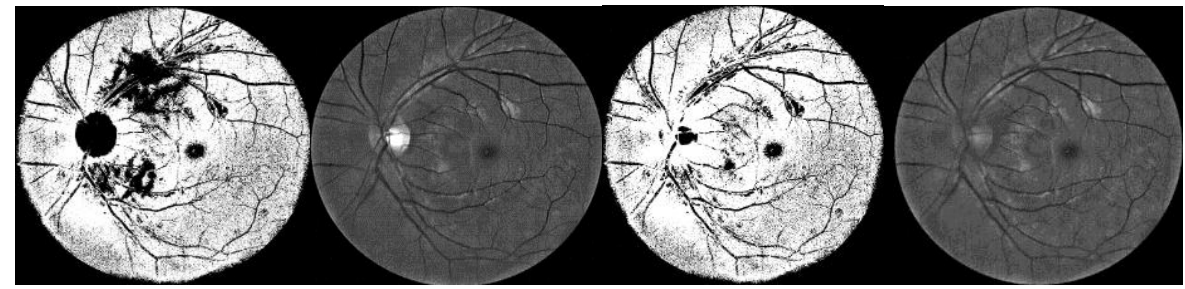

(d)

(e)

(f)

(g)

Fig. 2: (a) Non-uniform sampling grid, (b) Retinal image with uneven illumination and contrast, (c) First principal component of (b) from PCA analysis, (d) Background pixel classification using PCA analysis, (e) Image enhancement on single channel with PCA analysis, (f) Background pixel classification without the use of PCA analysis, (g) Image enhancement on single channel without the use of PCA. 


\subsection{Feature extraction}

\subsubsection{Adaptive weighted morphological functors}

All the previous generations of morphological operators apply some changing on the original image. However, they don't taking into account any information about the details and small information about the images and how they spread over the surface of the images. In other words, if there is a need to extract the small information in an image which is distributed in different directions, we need some tools which are able to adapt itself with the information in the image and extract the information from the original image with high accuracy. The previous generation of morphological operators dealing with such images, will extract only some parts of the information in specific directions as the knowledge of information distribution over the related area has not been considered.

In fact, structuring element used in adaptive weighted morphological functors (AWMF) [6], during scanning the original image, is updated and matches, step by step, with images direction that have the dominant information. In other words, the main aim of adaptive weighted morphological functors in our proposed algorithm is to produce continuous edge features from images under study. To start the process of extracting the retinal blood vessels, we use the improved and new generation of morphology functors introduced in this study, with automatic and adaptive weighted structure elements [6].

\subsubsection{Basic definitions}

In this section, foundations for a new generation of morphological edge detector based on the adaptive weighted morphological functors are explained. In this way, the edges available on the image along with their direction with the use of adaptive weighted structure elements and with high accuracy will be detected. The operation is in a way that, if the information exists in a specific direction, a large coefficient of the weight will be replaced in the structure element and otherwise a small weight factor will be replaced in the structuring element. Therefore, we can achieve a reinforced and revised edge detector. The aim of applying the adaptive weighted morphological functors is to produce the connected features from existing edges in the image under study.

The first step of implementing the adaptive weighted morphological functors is to search approximate directions of the edge in different parts of the image. To do so, we should first produce the initial edge image that contains the approximate information of the image under study. For this purpose, we use morphology gradient operator. 
where $X$ is the original image and $B$ is the initial structure element. $\oplus$ and $\bullet$ stand for the dilation and closing morphology operators, respectively. So this Edge Operator will be considered as the initial recognizer of the edges in the image and at the same time an adaptive selection of the final structure elements.

In the next step, we should locally search for the first recognized edges, and in the searching window intended for the final weighted structure elements. The proposed algorithm will use the edge research method presented in [7], named, "circumrotate cover". Also, the square window for the final weighted structure elements is used that allows the constructed edge in this structure elements rotate around the center. Then we will use the location of occurrence of the maximum variance of gray levels in this square window and we estimate locally the location of occurrence and the direction of the image edges in the first step. Now the weighted and normalized coefficient in final structure element will be selected based on the initial conditions of the edge. If the structure element be recognized to be belong to the edges, the coefficient of related pixels of the directional edge, will be 1 and the coefficient of further pixels will be determined by $w>1$. This will cause an emphasis on the effect of directional edge points and reduce the influence of the neighboring points. Other coefficients are made based on the Eq. (5).

$$
\Delta \omega=(\omega-1) / d
$$

Such that w is the sum of the coefficients related to edge in structure element under study and $d$ is the distance between the directional edge and the furthest spots from it. In horizontal and vertical directions, the weight of the components of the structure elements will reduce with $\Delta \omega$ parameter and each stage will start from the points belonging to the directional edge. A sample of adaptive weighted structure element of size $3 * 3$ and in different directions is shown in Fig. 3.

$$
A_{1}=\left[\begin{array}{ccc}
-1 & -1 & -1 \\
1 & 1 & 1 \\
-1 & -1 & -1
\end{array}\right] \quad, \quad A_{2}=\left[\begin{array}{ccc}
1 & 0 & -1 \\
0 & 1 & 0 \\
-1 & 0 & 1
\end{array}\right]
$$

Fig. 3: An example of adaptive weighted structure elements with the size of $3 * 3$. 
Finally, the weighted structure elements will be used to detect and extract the edge, as an adaptive weighted matrix for the detection and extraction of edges, with high accuracy and from digital images created from preprocessing stage. In this article, the modified TOPHAT function is used in order to extract the blood vessels from the background of retinal images. In Eq. (6), the modified TOPHAT is introduced.

TopHat $=I-\min \left(\left(I \bullet S_{C}\right) \circ S_{O} ; I\right)$

where $I$ is the original image and $S_{O}$ and $S_{C}$ are the structure element for opening and closing. Please note that in this article the adaptive weighted structure element is replaced for both structure elements. It is noteworthy that the operators being used in the modified TOPHAT function are of adaptive weighted morphological functors. In other words the introduced AWMF not only be able to extract the details of the image from the background in an effective way and improve the operation of morphological operators, also can combine gaps and accumulations on the final edge image and reduce their effectiveness.

The adaptive weighted morphological functors with the knowledge of the position and direction of the existed information in the image, starts the edge extraction from the images.

Due to adoptive and local operation, this functor can significantly increase the accuracy of extracting edge from image and at the same time avoid creating noise in the final edge image.

The equations related to weighted erosion morphology (WER) and weighted dilation morphology (WDI) are defined as:

$W E R(k, l)=\min _{u, v}\{X(k+u, l+v) / A(u, v)\}$

$W D I(k, l)=\max _{u, v}\{X(k+u, l+v) \times A(u, v)\}$

That $X$ is the image under study and $A$ is the adaptive weighted structure element. Other operators like weighted opening morphology (WOP) and weighted closing morphology (WCL) are a simple combination of WDI and WER defined as:

$W O P(X)=W D I(W E R(X))$

$W C L(X)=W E R(W D I(X))$ 


\subsection{Deleting frills}

In fact, this step is to label the connected components in the resulted binary edge images obtained from the previous step. In this step we have an image in which all connected blood vessels and arterioles have a specific label. For local processing, the labeled image is divided into the sub-images and then an adaptive and local threshold $T[8]$ is defined as:

$$
T=\mu-\alpha \sigma
$$

where $\sigma$ and $\mu$ are the adaptive parameters of above threshold and are the mean value and the standard deviation of length of the labels in each sub-image, respectively. The aforesaid filter acts so that it recognizes the labeled components whose the lengths are less than the local threshold $T$ as noise and removes them.

As some of the appeared frills have a same size as arterioles in the retinal images, we cannot use the connected component analysis (CCA) in one stage, because in addition to frills, some part of data will be removed from the image. Hence, we need two steps to remove the frills from the edge image. In the first step, to delete the frills smaller than arterioles, the method suggests the CCA with 4 neighborhoods and locally applies the introduced adaptive filter. Finally, by applying the connected component analysis with 8 neighborhoods of pixels and adaptive filter locally, the rest of frills will be removed and the image of blood vessels is extracted from the retinal image.

\section{EXPERIMENTAL RESULTS}

\subsection{Database}

This paper is applied to the well-known database DRIVE [9]. The 40 images which are in this database were selected from images related to 400 diabetic patients ranged between 50 and 90 years old in Netherlands. The images on this database are 584*565 pixels and in JPEG format. The set of 40 images has been divided into training and a test set, both containing 20 images. For the training images, a single manual segmentation of the vasculature is available. For the test cases, two manual segmentations are available; one is used as gold standard, the other one can be used to compare computer generated segmentations with those of an independent human observer. 


\subsection{Evaluation parameters}

Considering the segmented images by human in the DRIVE database, it is defined two following criteria in order to compare the results from algorithm with the results from segmentation by specialist:

- TP (True Positive): It indicates the pixels which algorithm recognized as blood vessels and in fact they are related to the blood vessels.

- FP (False Positive): It indicates the pixels which algorithm recognized as blood vessels but in fact they aren't related to the blood vessels.

- $\quad T N$ (True Negative): It indicates the pixels which algorithm not recognized as blood vessels and in fact, they aren't related to the blood vessels.

- $F N$ (False Negative): It indicates the pixels which algorithm not recognized as blood vessels but in fact, they are related to the blood vessels.

$T P R$ is the ratio of true positives to all pixels belonging to blood vessels; $F P R$ is the ratio of false positives to all pixels that do not belong to the blood vessels; TNR is the ratio of true negatives to all pixels that do not belong to the blood vessels, and FNR is the ratio of false negatives to all pixels belonging to blood vessels.

According to the definitions, accuracy of the algorithm is defined as:

$$
\text { Accuracy }=\frac{T P+T N}{T P+F N+T N+F P}
$$

\subsection{Pre-processing}

In order to improve the undesired illumination changes in retinal images and to smooth the illumination of such images, we consider $k_{1}$ and $k_{2}$ in Eq. (1) as $k_{1}=1.2$ and $k_{2}=1.5$, respectively. To determine the pixels in background and to estimate the illumination and contrast of the retinal images, we selected the size of the windows $w$ and $w_{1}$ as $W=70$ and $W_{1}=30$.

\subsection{Feature extraction}

In order to implement, the size of the structure element $B$ for the initial extraction of image's edges is considered $7 * 7$ and the size of the adaptive weighted structure elements $A$ for the final extraction of image's edges is considered $5 * 5$ so that they be not sensitive towards little changes and have the ability to extract the arterioles. 


\subsection{Deleting frills}

To remove the frills with the aid of the CCA analysis, we label the connected components of image in two stages, with 4 and 8 neighborhoods of pixels, respectively. Then, after dividing the image into blocks of $90 \times 90$ pixels in each stage, we apply the adaptive filter with the aforesaid threshold for all blocks, locally. The components, whose lengths are less than the specific threshold $T$, are recognized as noise and removed. In this algorithm, the value of the threshold is considered as $\mathrm{T}=15, \mathrm{~T}=30$ pixels, for two previously mentioned noise cancellation, respectively. Fig. 4 shows some results of blood vessel extraction in images from DRIVE database.
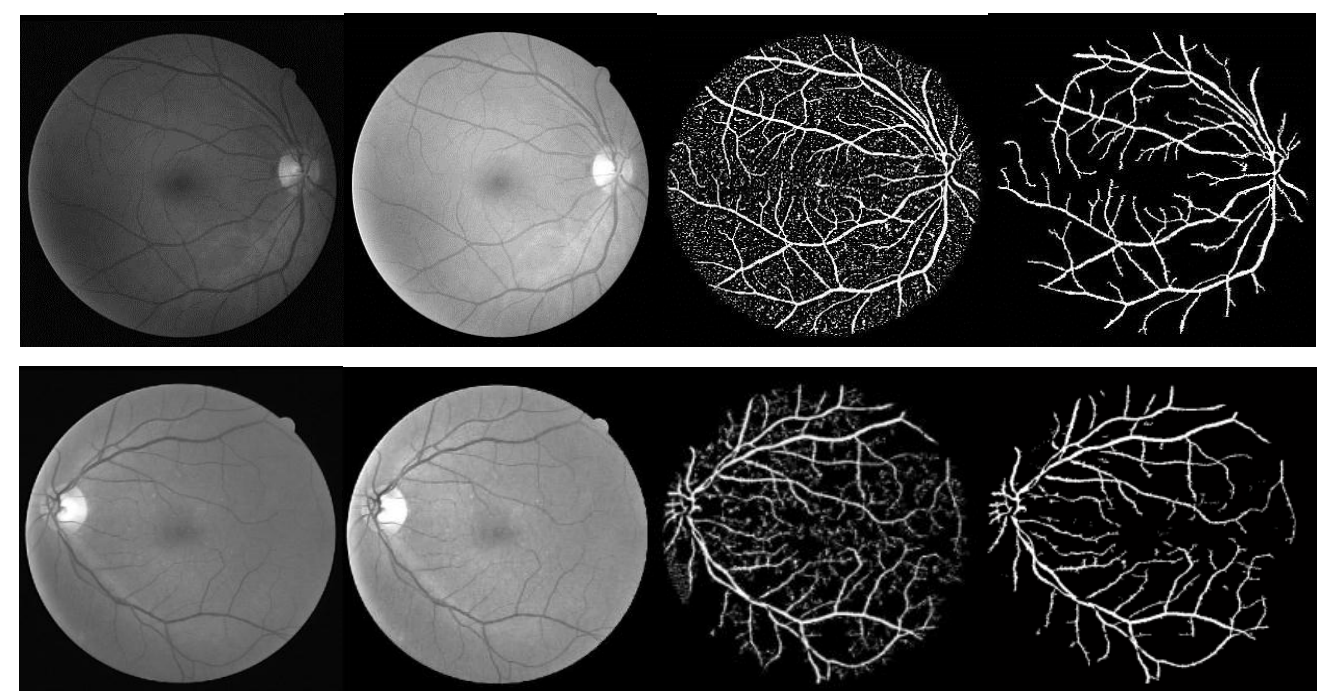

(a)

(b)

(c)

(d)

Fig. 4: (a) is the best sub band of original images, (b) is the enhanced images, (c) is the extracted blood vessels with frills, (d) is the final extracted blood vessels.

\subsection{Comparison with other algorithms}

Table 1 compares the performance of the proposed algorithm and some earlier reported methods based on DRIVE database in extracting the blood vessels from the retinal images. The proposed algorithm by A. M. Mendonca et al. [10], use mathematical morphology to benefit from a prioriknown vasculature shape features, such as being piecewise linear and connected. Then, by applying morphological operators, the vasculature is filtered from the background for the final segmentation. M. E. Martinez-Perez et al. [11] proposed a method based upon Multi-scale feature extraction. The local maxima over scales of the gradient magnitude and the maximum principal curvature of the Hessian tensor were used in a multiple pass region growing procedure. Growth progressively segmented the blood vessels by using both feature and spatial information. D. Marin et al. [12] 
presented a supervised method for blood vessel detection in digital retinal images. This method uses a neural network scheme for pixel classification and computes a 7-D vector composed of gray-level and moment invariants-based features for pixel representation.

In our algorithm, applying the pre-processing techniques, effectively omits the high intensity variations. Since the blood vessels are scattered in different directions, we used adaptive weighted structure elements for extracting blood vessels from retinal images. The use of AWMF in this manuscript causes to separate blood vessels from background with high accuracy. The results our implementation showes that the use of AWMF as the extractors of blood vessels from retinal images can significantly increase the accuracy of the algorithm. Finally, using the CCA analysis, effectively omits the created noise in the edge images.

Table 1: Comparison of blood vessels extraction algorithms

\begin{tabular}{|c|c|c|c|c|}
\hline Method & TPR & FPR & $\begin{array}{c}\text { Average } \\
\text { Accuracy } \\
(\%)\end{array}$ & $\begin{array}{c}\text { Sensitivity } \\
(\%)\end{array}$ \\
\hline $\begin{array}{c}\text { Mendonca } \\
\text { et al. [10] }\end{array}$ & 0.7344 & 0.0236 & $94.63 \pm 0.0065$ & 73.15 \\
\hline $\begin{array}{c}\text { Martinez-Perez et } \\
\text { al. [11] }\end{array}$ & 0.7246 & 0.0345 & 93.44 & 71.72 \\
\hline $\begin{array}{c}\text { Marin } \\
\text { et al. [12] }\end{array}$ & 0.7068 & 0.0305 & 94.52 & 70.67 \\
\hline Our method & 0.8875 & 0.0350 & $97.11 \pm 0.0093$ & $86.31 \pm 0.214$ \\
\hline
\end{tabular}

\section{CONCLUSION}

To optimal extraction of blood vessels from retinal images, for the first time, we used the adaptive weighted morphological functors in this article. unlike other edge detection operators, these functors during two stages will search for the edges on the surface of the image.

In the first stage and in order to find the approximate direction of the blood vessels in retinal images, we used ordinary morphological operators in this article. Then with new adaptive weighted structure elements according to the approximate direction of the edges found in the first stage, we attempted to re-extract the blood vessels in the final stage. In other words the adaptive weighted morphological 
functors and their structure elements that were introduced in this article, could purposefully look for the blood vessels in retinal images and could extracted them with high accuracy.

\section{REFERENCES}

[1] Wu Ch, Agam G, Stanchev P. A general framework for vessel segmentation in retinal images. In: Proc.of the IEEE Int. Sym. On Computational Intelligence in Robotics and Automation; 2007.

[2] Supot S, Thanapong Ch, Chuchart P, Manas S. Automatic segmentation of blood vessels in retinal image based on fuzzy kmedian clustering. In: Proc. Of the IEEE Int. Conf. on Integration Technology; 2007.

[3] Estabridis K, Defigueiredo R. Blood vessel detection via a multi window parameter transform. In: Proc. of the 19th IEEE Symp. on Computer-Based Med. Sys. (CBMS'06); 2006.

[4] Joshi GD, Sivaswamy J. Colour retinal image enhancement based on domain knowledge. In: 6th Indian Conf. on Computer Vision, Graphics \& Image Processing; 2008.

[5] Shahbeig S, Pourghassem H. Fast and automatic algorithm for optic disc extraction in retinal images using principle-component-analysis-based preprocessing and curvelet transform. Journal of the Optical Society Of America A-Optics Image Science and Vision 2013; 30(1): 13-21.

[6] Sedaaghi MH, Wu QH. Weighted morphological filter. Electronics Letters Online 1998; 34(16): 1566-1567.

[7] Heath M, Sarkar S, Sanocki T, Bowyer K. Comparison of edge detectors: a methodology and initial study. Computer Vision and Image Understanding 1998; 69(1): 38-54.

[8] Hamadani N. Automatic target cueing in IR imagery. Master's thesis, Air Force Institute of Technology, WPAFB, Ohio; 1981.

[9] Images from DRIVE database are available at: http://www.isi.uu.nl/Research/Databases/DRIVE/

[10] Mendonça AM, Campilho A. Segmentation of retinal blood vessels by combining the detection of centerlines and morphological reconstruction. IEEE Trans. Med. Imag. 2006; 25(9): $1200-1213$.

[11] Martinez-Perez ME, Hughes AD, Thom SA, Bharath AA, Parker KH. Segmentation of blood vessels from red-free and fluorescein retinal images. Med. Imag. Anal. 2007; 11(1): 47-61.

[12] Marín D, Aquino A, Gegúndez-Arias ME, Bravo JM. A new supervised method for blood vessel segmentation in retinal images by using gray-level and moment invariantsbased features. IEEE Trans. Med. Imag. 2011; 30(1): 146-158. 